\title{
Formation of Millisecond Pulsars in Globular Clusters
}

\author{
Natalia Ivanova* ${ }^{*}$ Craig O. Heinke ${ }^{\dagger, * *}$ and Frederic A. Rasio** \\ *CITA, University of Toronto, 60 St George St, Toronto, ON M5S 3H8, Canada \\ ${ }^{\dagger}$ Department of Astronomy, University of Virginia, 530 McCormick Road Charlottesville, VA 22904-4325, USA \\ ${ }^{* *}$ Physics and Astronomy Department, Northwestern University, 2145 Sheridan Rd, Evanston, IL 60208 USA
}

\begin{abstract}
In this contribution we discuss how neutron stars are produced and retained in globular clusters, outlining the most important dynamical channels and evolutionary events that affect the population of mass-transferring binaries with neutron stars and result in the formation of recycled pulsars. We confirm the importance of electron-capture supernovae in globular clusters as the major supplier of retained neutron stars. By comparing the observed millisecond pulsar population and the results obtained from simulations, we discuss several constraints on the evolution of mass-transferring systems. In particular, we find that in our cluster model the following mass-gaining events create populations of MSPs that do not match the observations (with respect to binary periods and companion masses or the number of produced systems) and therefore likely do not lead to NSs spun up to millisecond periods: (i) accretion during a common envelope event with a NS formed through accretion-induced collapse, and (ii) mass transfer from a WD donor. By restricting ourselves to the evolutionary and dynamical paths that most likely lead to neutron star recycling, we obtain good agreement between our models and the numbers and characteristics of observed millisecond pulsars in the clusters Terzan 5 and 47 Tuc.
\end{abstract}

Keywords: pulsars, neutron stars, globular clusters

PACS: 97.60.Gb, 97.60.Jd, 97.80.-d, 97.80.Jp, 98.70.Qy, 98.20.Gm, 98.20.jP

\section{INTRODUCTION}

Millisecond pulsars (MSPs) are present in globular clusters (GCs) in great numbers: about 140 GC MSPs have been detected. with more than a dozen in several GCs - in 47 Tuc [1], M28 [2], and Terzan 5 [3]. It is estimated that of order 1000 potentially detectable MSPs are present in the Galactic GC system [4], as at present the pulsar searches have reached the bottom of the pulsar luminosity function only in a few clusters (e.g., 47 Tuc and M15).

Per unit mass, the number of MSPs in GCs greatly exceeds their numbers in the Galaxy. This was, however, an expected discovery: MSPs are thought to be descendants of LMXBs (for a review see, e.g., Bhattacharya and van den Heuvel 1991), and the abundance of X-ray binaries per unit mass was known to be $\sim 100$ times greater in GCs than in the Galaxy as a whole [5]. The high formation rate of X-ray binaries, in itself, is widely accepted to be a consequence of the high stellar density of GCs, which may lead to the creation of compact NS binaries in close stellar encounters.

Although the numbers of MSPs and X-ray binaries per unit mass in globulars are much higher than those in the rest of the Galaxy, only a few X-ray binaries or a few dozen MSPs are present per fairly massive (more than a million stars) and dense cluster. This makes the problem

${ }^{1}$ See http://www.naic.edu $\sim$ pfreire/GCpsr.html for an updated list. computationally very challenging. E.g., the target time for direct $N$-body methods to address the million-body problem is as far in the future as 2020 (Hut 2006). In our studies, we use a modified encounter rate technique method, described in detail in Ivanova et al. [6], and with the updates described in Ivanova et al. [7].

\section{NEUTRON STAR PRODUCTION}

In our studies we adopt that a NS can be formed as a result of either a core-collapse (CC) supernova, which occurs after an iron core is formed, or an electron capture supernova (ECS). The latter, it has been argued, occurs when a degenerate $\mathrm{ONeMg}$ core reaches $M_{\mathrm{ecs}}=1.38 M_{\odot}$; its collapse then is triggered by electron capture on ${ }^{24} \mathrm{Mg}$ and ${ }^{20} \mathrm{Ne}$ before neon and subsequent nuclear burnings start, and therefore before iron core formation $[8,9,10$, 11, 12].

There are several possible situations when a degenerate $\mathrm{ONeMg}$ core can be developed and reach $M_{\mathrm{ecs}}$ :

- Evolutionary induced collapse (hereafter EIC).

If the initial core mass is less than required for neon ignition, $1.37 M_{\odot}$, the core becomes strongly degenerate and grows to $M_{\text {ecs }}$ through the continuing $\mathrm{He}$ shell burning. The critical mass range for this to occur in single stars is somewhere between 6 and 10 $M_{\odot}$. In more massive stars, carbon, oxygen, neon and silicon burnings progress under non-degenerate conditions, and, in less massive stars, $\mathrm{ONeMg}$ cores 
TABLE 1. Production of NSs.

\begin{tabular}{llccc}
\hline & CC & EIC & AIC & MIC \\
\hline single & & & & \\
$Z=0.0005$ & 3354 & 594 & - & - \\
$Z=0.001$ & 3255 & 581 & - & - \\
$Z=0.005$ & 2833 & 570 & - & - \\
$Z=0.02$ & 2666 & 400 & - & - \\
binary & & & & \\
$Z=0.0005$ & 3079 & 545 & 59 & 14 \\
$Z=0.001$ & 3056 & 553 & 60 & 15 \\
$Z=0.005$ & 2750 & 576 & 58 & 16 \\
$Z=0.02$ & 2463 & 406 & 33 & 20 \\
\hline
\end{tabular}

* Production of NSs in the case of no dynamics. Notations for channels - CC - core-collapse supernova; EIC - evolution-induced collapse; AIC - accretion induced collapse; MIC - mergerinduced collapse. Numbers are scaled per 200,000 $M_{\odot}$ stellar population mass at the age of $11 \mathrm{Gyr}$.

never form. This critical mass range depends on the properties of the $\mathrm{He}$ and $\mathrm{CO}$ cores, which, in turn, are highly dependent on the mixing prescription (semiconvection, overshooting, rotational mixing, etc.) and varies between different evolutionary codes [see discussion in 13, 14]. In the code that we use for our cluster simulations, a non-degenerate $\mathrm{ONeMg}$ core is formed when the initial He core mass is about $2.25 M_{\odot}[15,16]$ and the ranges of initial masses for single stars that lead to the formation of such a core are 6.85 to $7.57 M_{\odot}$ and 6.17 to $6.76 M_{\odot}$ for metal-rich GCs $(Z=0.005)$ and metalpoor GCs $(Z=0.0005)$, respectively. In binaries, the mass transfer history of the star may affect the range of progenitor masses for which an ECS can occur [13]. We find that in our simulations the mass range can extend to as low as $3 M_{\odot}$ and to as high as $22 M_{\odot}$.

- Accretion induced collapse (hereafter AIC). AIC can occur by accretion on to a degenerate $\mathrm{ONeMg}$ WD in a binary, where such a WD steadily accumulates mass until it reaches the critical mass $M_{\text {ecs }}$. The set of conditions for the MT rates which allow mass accumulation can be found in Ivanova and Taam [17].

- Merger induced collapse (hereafter MIC). When two WDs coalesce and the product has a total mass exceeding $M_{\text {ecs }}$, either one of the WDs is a massive ONeMG WD or the two are both $\mathrm{CO}$ WDs. In the latter case, an off-centre carbon ignition converts the coalesced star into an ONeMg core, and then the WD proceeds with an ECS as usual [18, 19].

The gravitational mass of the newly formed NS is $\sim 0.9$ of its baryonic mass and will be $1.26 M_{\odot}$ in the case of AIC or EIC. However, in the case of MIC, it is possible to form a more massive NS. The reason is that the condition for ECS to occur depends on the central density. As a consequence, the collapse of a rapidly rotating WD, which was formed during the coalescence of two WDs with total mass exceeding the Chandrasekhar mass, can lead to the formation of a more massive, and also rapidly spinning, NS.

In Table 1 we show the results for NS production via different channels in stellar populations of several metallicities - from solar (as in the Galactic field) to that of a typical metal-poor cluster: $\mathrm{Z}=0.02,0.005,0.001,0.0005$. Note that the production of NSs via core-collapse $\mathrm{SNe}$ (CC NSs), per unit of total stellar mass at $11 \mathrm{Gyr}$, decreases as metallicity increases. The difference between the solar metallicity case and the metal-poor case is about 20 percent, while the difference between metal-rich and metal-poor clusters $(Z=0.005$ and $Z=0.0005)$ is about 10 15 percent.

EIC in single stars for metal-rich populations occurs in stars of higher masses. The range of initial masses is, however, the same for both populations and is about $0.6 M_{\odot}$. As a result, in agreement with the adopted Kroupa power law for the IMF [20]. the number of NSs produced via EIC (EIC NSs) from the population of single stars is smaller in the case of a Galactic field population than in the case of a GC population by 30 per cent, while the difference between metal-rich and metal-poor clusters is only 5 per cent.

AIC and MIC NSs can be produced only in populations with binaries. The range of initial masses of progenitors is not as strongly defined as for EIC in single stars. As a result, their production rates do not have a strong dependence on the metallicity.

\section{NEUTRON STAR RETENTION}

To estimate how many NSs can be retained in GCs, we first considered stellar populations without dynamics. In our simulations, we adopt the most recently derived pulsar kick velocity distribution from [21]. It is a Maxwellian distribution with one-dimensional RMS velocity $\sigma=265 \mathrm{~km} \mathrm{~s}^{-1}$. For comparison, we also considered a case with the natal kick distribution as in earlier studies [22] (with $\mathrm{Z}=0.005$, typical for a metal-rich globular cluster). There, the kick velocity distribution is 2 Maxwellians with a lower peak for kick velocities at 90 $\mathrm{km} / \mathrm{s}$.

For ECS NSs from all the channels (EIC, AIC and MIC), we adopt that the accompanying natal kick is 10 times smaller: we adopt the results of numerical simulations which find that the SASI instability, required by current understanding for the large explosion asymmetry in the case of core-collapse supernovae, fails to develop [23, 24]). As a result, natal kicks for ECS NSs do not 


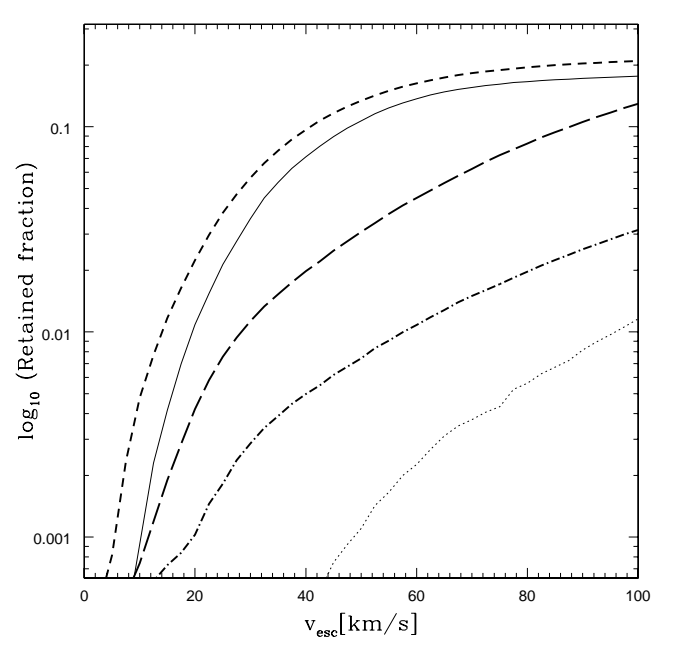

FIGURE 1. The retention fractions as a function of escape velocity (for stellar evolution unaffected by dynamics) for a [21] kick distribution. Dotted and dash-dotted lines show the retention fractions for single and binary populations, corecollapse NSs only. Solid and short-dashed lines show the total retention fractions for single and binary populations, all NSs. For comparison, we show the total retention fraction of a binary population with the [22] kick distribution (long-dashed line).

exceed $100 \mathrm{~km} / \mathrm{s}$.

We find that even considering a stellar population with $100 \%$ primordial binaries, the retention fraction of CC NSs is very small (Fig. 11); the resulting number of retained CC NSs is just a few per typical dense globular cluster of $2 \times 10^{5} M_{\odot}$. In contrast, NSs formed via different ECSs channels provide about 200 retained NSs per typical GC (similar numbers were found also in [25]). Therefore, in contrast to the population of NSs in the Galaxy, the population of NSs in GCs is mainly lowmass NSs made by ECS. With the old distribution for natal kick velocities, reasonable numbers of NSs can be produced and retained in a typical GC, while a massive cluster like 47 Tuc could retain as many as 600 NSs (see also Fig. 11). This result agrees with the retention fractions obtained in Pfahl et al. [26].

In the case when dynamical encounters affect the stellar population, and, as an example, could destroy primordial binaries, the resulting numbers of produced and retained NSs are somewhere between only-primordialsingle star case and only-primordial-binary case. The exceptions are AIC and MIC NSs, the formation of which is enhanced by dynamical encounters. Overall, a typical dense cluster will retain 8 CC NSs, $\sim 170$ EIC NSs, $\sim 50$ AIC NSs and $\sim 20$ MIC NSs; Terzan 5 will retain in total about 500 NSs (assuming that the cluster mass is 370 $000 M_{\odot}$ ) and about 1100 NSs will be in a cluster like 47 Tuc (if its total mass is $10^{6} M_{\odot}$ ).

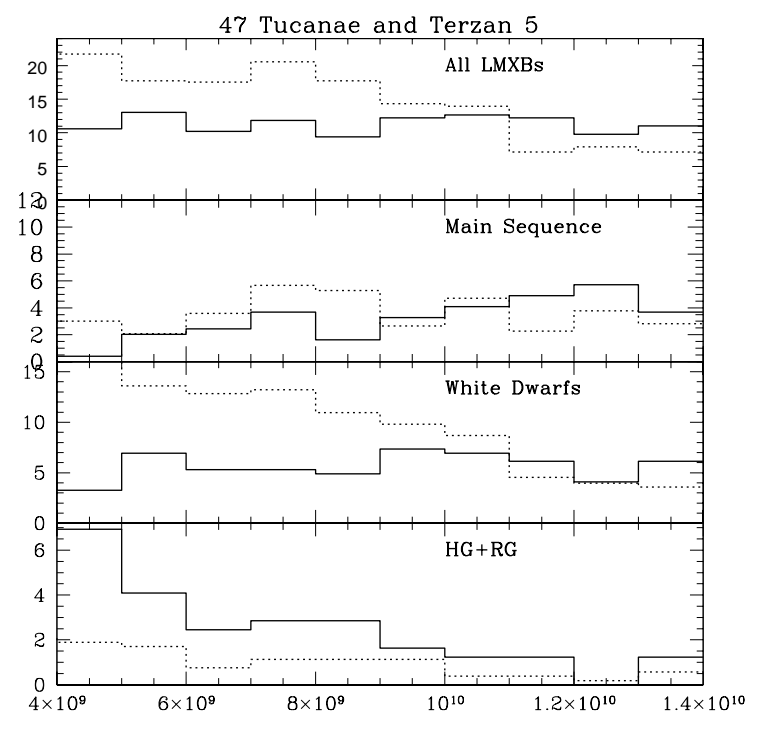

FIGURE 2. Number of appearing LMXBs per Gyr with different donor types (with all donors, with main sequence donors, with white dwarf donors and with donors that are red giants or subgiants or are in the Hertzsprung Gap) for 47 Tuc (solid line) and Terzan 5 (dotted line).

\section{NEUTRON STAR RECYCLING}

A common understanding of MSP formation is that the NS is recycled through disk accretion. The formation rate of LMXBs can therefore be linked with the number of observable MSPs. The connection between the formation rate of LMXBs and their existence at a give time in a $\mathrm{GC}$ is provided by the life-time $\tau_{\mathrm{LMXB}}$ of an LMXB with a particular donor. An average $\tau_{\mathrm{LMXB}}$ for NS-MS LMXBs is about 1 Gyr. Depending on the metallicity and the initial donor mass, a system can be persistent 5-40 percent of the MT time for metal-rich donors > $0.6 M_{\odot}$ and transient at the rest; for donors of lower metallicities or smaller masses, a NS-MS LMXB will be transient all the time [27], and therefore most likely be seen as a quiescent LMXB (qLMXB) rather than a bright LMXB. In the case of NS-WD LMXBs (ultracompact X-ray binaries, UCXBs), total $\tau_{\mathrm{LMXB}}$ is few Gyr, however the time when a system is persistent and has an X-ray luminosity above $10^{36} \mathrm{erg} / \mathrm{s}$ is $10^{7} \div 10^{8}$ yr only. An LMXB with a red giant companion or a companion that is in the Hertzsprung gap is very shortlived, $10^{5}-10^{7} \mathrm{yr}$, and in only very rare cases can they live as long as $10^{8} \mathrm{yr}$.

In our simulations, we find that a typical GC can contain up to 2 LMXBs with a MS companion (most likely observed, at any particular time, as qLMXBs) and up to one LMXB with a WD companion (ultra-compact X-ray 
binaries, UCXBs). The scatter in the average number of observed LMXBs per cluster in independent simulations is rather large (more than expected from Poisson statistics) - e.g., for UCXBs, it can vary between 0.1 and 1.1. In the case of Terzan 5 and 47 Tucanae, the average number of LMXBs formed per Gyr, at the age of $11 \mathrm{Gyr}$, is $\sim 5$ for NS-MS LMXBs and $\sim 8$ for UCXBs (see Fig. (2). These numbers are in general agreement with the observations: (i) assuming that $\tau_{\mathrm{UCXB}} \sim 10^{8} \mathrm{yr}$, those numbers are consistent with the presence of one bright UCXB in Terzan 5 and no bright UCXB detected in 47 Tuc; (ii) several qLMXBs are identified in both Terzan 5 and 47 Tuc.

Overall the numbers of NSs that gain mass via mass transfer (MT) through $11 \mathrm{Gyr}$ of cluster evolution are high: for our 47 Tuc model, about 50 NS-MS binaries and about 100 UCXBs. As we observe fewer MSPs in these GCs, while the rate of LMXB formation in simulations is consistent with the observations, we conclude that not all NSs that gain mass via MT become currently active MSPs.

Indeed, it has been argued that a NS is spun up only if the accretion rate is not too low, $\dot{M} \geq 3 \times 10^{-3} \dot{M}_{\text {Edd }}$, where $\dot{M}_{\text {Edd }}$ is the Eddington limit (for a review see, e.g., Lamb \& Yu 2005). In a UCXB, soon after the start of mass transfer, the accretion rate drops very quickly. After $1 \mathrm{Gyr}$, it is less than $10^{-4} \dot{M}_{\text {Edd. }}$ Such a MT leads to a spin-down of the previously spun-up NS, and no MSP is formed. Support for this statement is given by the fact that no UCXBs (those that have WD companions) are visible as radio MSPs (Lamb \& Yu 2005).

The requirement of steady spin-up through disk accretion implies that not all physical collisions will lead necessarily to NS spin-up. In the case of a physical collision with a giant, the NS will retain a fraction of the giant envelope, with a mass of a few hundredths of $M_{\odot}$ (Lombardi et al. 2006). Immediately after the collision, this material has angular momentum and most likely will form a disk. We adopted therefore that in the case of a physical collision with a giant, an MSP can be formed, but, in the case of any other physical collision, the NS will not be recycled.

\section{MILLISECOND PULSARS}

Suppose that all mass-gaining events in the life of a NS - mass transfer, physical collision with a red giant, common envelope hyper-accretion or merger - can lead to NS recycling. In this case we found that as many as 250 and 320 potential MSPs are made in our simulations of clusters like Terzan 5 or 47 Tuc, respectively (the corresponding numbers of retained NSs are $\sim 500$ and $\sim 1100$ ). Although these numbers correlate well with the formation rate of LMXBs, they greatly exceed the numbers of observed and inferred MSPs in both clusters, which are 33 (in Terzan 5; perhaps 60 total) and 22 (in 47 Tucanae; perhaps 30 total).

By considering the whole population of NSs that gained mass, we found that bMSPs formed from primordial binaries, where a common envelope event led to AIC, create a population of potential bMSPs with relatively heavy companions, in circular orbits with periods from one day to several hundreds of days. This population is not seen in either Terzan 5 or 47 Tuc. We considered primordial binaries that evolved through mass transfer from a giant donor after a NS was formed via AIC. Even though bMSPs that have similar periods, companion masses and eccentricities are present in Terzan 5, there are no such systems in 47 Tucanae. Also, bMSPs made from primordial binaries after AIC must inevitably be formed in low-dense clusters, but no bMSPs are observed there. These facts tell us that either AIC does not work, or the kicks in the case of AIC are stronger then we adopted, or a NS formed via AIC has such a strong magnetic field, that surface accretion does not occur.

Let us summarize the mass-gain scenarios described above (in this and the previous Section) which might not lead to the formation of MSPs:

- Primordial bMSPs where a NS gained mass via CE

- bMSPs where a NS gained mass via merger

- Mass transferring NS-MS systems with a donor mass above $\sim 0.05-0.1 M_{\odot}$, as they most likely are seen only as LMXBs/qLMXBs

- Mass transfer in NS-WD systems does not produce millisecond pulsars

Considering all the exclusions described above, we form in our simulations at least $15 \pm 7$ MSPs for Terzan 5 and $25 \pm 4$ MSPs for 47 Tuc (for the formed population of bMSPs, see Fig. 3). The values for Terzan 5 are somewhat uncertain due to uncertainty in the properties of this heavily reddened cluster. The binary population of MSPs is $1 / 2-1 / 3$ of all MSPs. The total number of NSs that gain mass in the simulations are 250 and 320 in Terzan 5 or 47 Tuc, accordingly.

We predict that the fraction of single pulsars is higher in Terzan 5 than in 47 Tuc, as observed. The origin of isolated MSPs depends on the cluster dynamical properties. For example, in our standard model, about half of isolated MSPs were formed in a result of an evolutionary merger at the end of the MT from a MS companion, and another half lost their companion as a result of a binary encounter. Most such binary encounters occurred in systems where a NS was spun-up during MT from a giant, and had a WD companion just before it become single. In the case of 47 Tuc, very few isolated pulsars were formed in a result of an evolutionary merger at the end of the MT. Most lost their companion as a result of a 

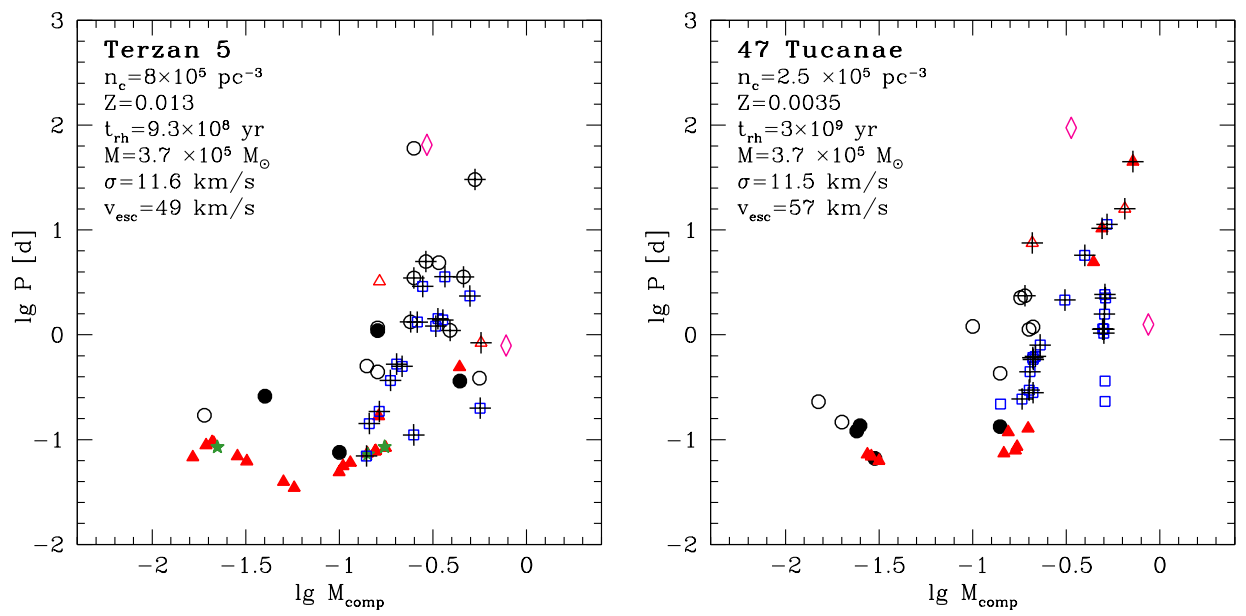

FIGURE 3. bMSPs in simulated models of 47 Tuc and Terzan 5 compared to observed bMSPs. The simulated populations correspond to several independent runs and represent a larger population than in the observed clusters. Observed bMSPs are shown with circles; triangles - bMSP formed via binary exchanges; stars - via tidal captures; squares - via physical collisions; diamonds - primordial binaries. Cross signs mark eccentric bMSPs $(e \geq 0.05)$ and solid symbols mark systems with a non-degenerate companion (in the case of simulations) or observed eclipsing systems.

binary encounter, where in half of the cases the companion before the binary destruction was a low-mass WD or MS star at the end of their MT sequence.

MSPs are likely to be located where they were formed. Most of them are in the core and less than a third in the halo. Halo MSPs cam be primordial or recoiled, where the fraction of recoiled MSPs increases as $v_{\text {rec }}$ decreases. Roughly half of observed pulsars are found outside their cluster core radius [28], but the radial distribution of most pulsars is exactly as expected for a population that is produced in or around the core [29, 4], with the exception of a few, likely ejected, pulsars in M15 and NGC 6752 [e.g. 30].

\section{DISCUSSION}

We considered in detail the problem of the formation of MSPs in GCs, which includes NS formation, retention and recycling. Our simulations showed that most of the retained neutron stars in GCs must come from an electron capture supernova formation channel (c.f. the field, where most NSs are from core-collapse supernovae). A typical GC could contain at present (where the adopted cluster age is $11 \mathrm{Gyr}$ ) as many as $\sim 200 \mathrm{NSs}$ where about half of them are located outside the core; a massive GC like 47 Tuc could have more than a thousand NSs.

Our simulations produce LMXBs in numbers comparable to observations if indeed as many as a few hundred NSs are retained per GC. If, contrary to our assumptions, AIC does not lead to the formation of NSs, then the number of formed NSs is reduced only by $\sim 20 \%$, but the number of appearing LMXBs is decreased by 2-3 times (per Gyr, at the cluster age of $11 \mathrm{Gyr}$ ). Given the large scatter in the simulations, it may still be consistent with the observations.

We find that, in case of a very dense cluster, up to half of NSs could gain mass after their formation through mass transfer, hyper-accretion during a common envelope, or physical collision. It is likely that most of these mass-gaining events do not lead to NS spin-up, and that only a few percent of all NSs appear eventually as MSPs, implying that there is a large underlying population of unseen NSs in GCs. In particular, we find that if we assume that a NS would accrete and spin up during a common envelope, we overproduce bMSPs in the cluster halos from primordial binaries of intermediate masses. Such bMSPs would be present in low-density clusters and have not yet been seen.

Excluding the systems discussed above, as well as those which are still actively accreting their donor's material and are seen instead as LMXBs, we obtained the number of "detectable" bMSPs. The predicted numbers about 2 dozen - are in good agreement with the observations. The fraction of isolated pulsars is comparable with that observed and is bigger in Terzan 5 than in 47 Tuc. Comparing the population census of our models with the observations of all detected pulsars to date in GCs, we find good agreement for all types of pulsars.

In conclusion we outline several important questions that must be addressed for further progress in studies of NSs in globular clusters:

- What is the final fate of a mass-transferring NS-WD binary - the post-MT period, the NS luminosity in 
its quiescent state, and how does it lose its companion?

- What is the result of mass accretion on a NS during a merger or a physical collision?

- For a common envelope event in primordial binaries of intermediate masses that produce a NS via ECS does the NS accrete the material and spin up? What is the common envelope efficiency?

\section{REFERENCES}

1. F. Camilo, D. R. Lorimer, P. Freire, A. G. Lyne, and R. N. Manchester, ApJ 535, 975-990 (2000).

2. I. H. Stairs, S. Begin, S. Ransom, P. Freire, J. Hessels, J. Katz, V. Kaspi, and F. Camilo, "New Pulsars in the Globular Cluster M28," in American Astronomical Society Meeting Abstracts, 2006, pp. 159.02-+.

3. S. M. Ransom, J. W. T. Hessels, I. H. Stairs, P. C. C. Freire, F. Camilo, V. M. Kaspi, and D. L. Kaplan, Science 307, 892-896 (2005), astro-ph/0501230

4. C. O. Heinke, J. E. Grindlay, P. D. Edmonds, H. N. Cohn, P. M. Lugger, F. Camilo, S. Bogdanov, and P. C. Freire, ApJ 625, 796-824 (2005).

5. G. W. Clark, ApJL 199, L143-L145 (1975).

6. N. Ivanova, K. Belczynski, J. M. Fregeau, and F. A. Rasio, MNRAS 358, 572-584 (2005).

7. N. Ivanova, C. Heinke, F. A. Rasio, K. Belczynski, and J. Fregeau, ArXiv e-prints 706 (2007), 0706.4096.

8. S. Miyaji, K. Nomoto, K. Yokoi, and D. Sugimoto, PASJ 32, 303-+ (1980).

9. K. Nomoto, ApJ 277, 791-805 (1984).

10. K. Nomoto, ApJ 322, 206-214 (1987).

11. F. X. Timmes, and S. E. Woosley, ApJ 396, 649-667 (1992).

12. F. X. Timmes, S. E. Woosley, and R. E. Taam, ApJ 420, 348-363 (1994).

13. P. Podsiadlowski, N. Langer, A. J. T. Poelarends, S. Rappaport, A. Heger, and E. Pfahl, ApJ 612, 1044-1051 (2004).

14. L. Siess, $A \& A$ 448, 717-729 (2006).

15. O. R. Pols, K.-P. Schroder, J. R. Hurley, C. A. Tout, and P. P. Eggleton, MNRAS 298, 525-536 (1998).

16. J. R. Hurley, O. R. Pols, and C. A. Tout, MNRAS 315, 543-569 (2000).

17. N. Ivanova, and R. E. Taam, ApJ 601, 1058-1066 (2004).

18. H. Saio, and K. Nomoto, $A \& A$ 150, L21-L23 (1985).

19. H. Saio, and K. Nomoto, ApJ 615, 444-449 (2004).

20. P. Kroupa, Science 295, 82-91 (2002).

21. G. Hobbs, D. R. Lorimer, A. G. Lyne, and M. Kramer, MNRAS 360, 974-992 (2005).

22. Z. Arzoumanian, D. F. Chernoff, and J. M. Cordes, ApJ 568, 289-301 (2002).

23. R. Buras, H.-T. Janka, M. Rampp, and K. Kifonidis, $A \& A$ 457, 281-308 (2006), arXiv: astro-ph/0512189.

24. F. S. Kitaura, H.-T. Janka, and W. Hillebrandt, $A \& A$ 450, 345-350 (2006), arXiv:astro-ph/0512065

25. A. G. Kuranov, and K. A. Postnov, Astronomy Letters 32, 393-405 (2006), astro-ph/0605115

26. E. Pfahl, S. Rappaport, and P. Podsiadlowski, ApJ 573, 283-305 (2002).
27. N. Ivanova, ApJ 636, 979-984 (2006).

28. F. Camilo, and F. A. Rasio, "Pulsars in Globular Clusters," in ASP Conf. Ser. 328: Binary Radio Pulsars, edited by F. A. Rasio, and I. H. Stairs, 2005, pp. 147-+.

29. J. E. Grindlay, F. Camilo, C. O. Heinke, P. D. Edmonds, H. Cohn, and P. Lugger, ApJ 581, 470-484 (2002).

30. M. Colpi, A. Possenti, and A. Gualandris, ApJL 570, L85-L88 (2002), astro-ph/0204291. 